\title{
Cambios en los parámetros de calidad del trigo candeal durante el almacenamiento
}

\author{
Astiz,V., Errea, E. A., Molfese, E. R. y Seghezzo, M. L.
}

\section{RESUMEN}

La calidad del trigo recién cosechado y las fluctuaciones que puede sufrir durante el almacenamiento resultan temas de interés en la industria molinera. El objetivo de este trabajo fue estudiar los cambios producidos en la calidad industrial del trigo candeal (Triticum durum L.) recién cosechado y durante siete meses de almacenamiento. Los parámetros de grano y sémola determinados fueron: peso hectolitro, peso de mil granos, proteína, humedad, rendimiento molinero, falling number, color, contenido de gluten húmedo y seco, índice de gluten y propiedades farinográficas. La humedad del grano se mantuvo por debajo del $14 \%$ durante el tiempo de almacenamiento. Se observaron fluctuaciones en las tasas de extracción de sémola durante los primeros meses de ensayo. Los valores de extracción variaron desde el 66 al $73 \%$, estabilizándose en $72 \%$ a los 120 días de la cosecha. El falling number también presentó una tendencia creciente. El resto de los parámetros de calidad, incluyendo gluten y farinograma, no mostraron variaciones significativas durante el almacenamiento. Los resultados indican que no hay evidencia de cambios en los parámetros de calidad, excepto en la extracción de sémola. Esto sugiere el efecto positivo de mezclar trigo viejo con trigo nuevo para minimizar fluctuaciones en los parámetros de molienda.

Palabras clave: trigo candeal, almacenamiento, calidad industrial.

Astiz,V., Errea, E. A., Molfese, E. R. and Seghezzo, M. L. , 2017. Changes in durum wheat quality parameters during storage. Agriscientia 34 (II): 25-32

\section{SUMMARY}

The quality of newly harvested wheat and the changes that may occur during grain storage is of interest to the milling industry. The objective of this work was to study the variations in the industrial quality of durum wheat from the moment of its harvest throughout 7 months of storage. Grain and semolina parameters such as hectoliter weight, one thousand kernel weight, protein, moisture, milling yield, falling number, color, wet and dry gluten content, gluten index and 
farinograph properties were determined. Grain moisture was below 14\% during storage time. Fluctuations of semolina extraction rates during the first months of storage were observed. Extraction values varied from 66 to $73 \%$, stabilizing in $72 \%$ at 120 days from harvest. The falling number also presented an increasing tendency. The other quality parameters including gluten and farinograph, showed no significant variations during the storage time. The results indicate that there is no evidence of changes in quality parameters except for milling yield. This suggests the positive effect of blending old wheat with new wheat to minimize fluctuations in milling parameters.

Key words: durum wheat, storage, industrial quality.

V. Astiz, E. A. Errea, E. R. Molfese y M. L. Seghezzo: Laboratorio de Calidad Industrial de Granos, Chacra Experimental Integrada Barrow (Convenio INTA-MAI), RN No $3 \mathrm{~km}$ 487, CC 50, provincia de Buenos Aires, Argentina. Correspondencia a: valentina@inta.gob.ar

\section{INTRODUCCIÓN}

En el mundo se cultivan unas 18,5 millones de hectáreas con trigo candeal, que producen 35-40 millones de toneladas y representan un $8 \%$ de la producción mundial de trigo. Argentina alcanzó el tercer lugar en el mundo como país exportador al obtener una producción de 760000 tn en la campaña1969-1970 (Seghezzo, 2014). En la última campaña (2016-2017) se produjeron 162426 tn, según datos del Ministerio de Agroindustria de la Nación (2017).

En Argentina, el cultivo de trigo candeal se encuentra localizado principalmente en el sudestecentro sur y oeste de la provincia de Buenos Aires. De ahí la importancia que la Chacra Experimental Integrada Barrow le da a este cultivo (Seghezzo, 2014).

Los cereales son sometidos a almacenamiento durante períodos relativamente largos. Si se cosechan con contenidos de humedad bajos, se guardan protegidos de las inclemencias meteorológicas y evitando insectos y roedores, se pueden conservar fácilmente. El sistema más generalizado es el almacenamiento a granel.

El objetivo de la industria molinera es proveer harinas o sémolas de una calidad constante que cumpla los requerimientos de sus clientes. La calidad tecnológica del trigo puede sufrir modificaciones asociadas al tiempo de almacenamiento y a las condiciones imperantes en el silo: temperatura, humedad y concentración de oxígeno (Gras y O'Riordan, 1998). Químicos cerealeros y consumidores de harina han reconocido por muchos años que el comportamiento molinero de una harina mejora con el tiempo (Swanson, 1931, 1946; Pomeranz, 1978) hasta un punto en que la calidad se estabiliza y, por un período largo, solo se producen cambios muy pequeños.

En el ambiente de la molinería se asegura que el trigo no debe ser procesado hasta que haya transcurrido por lo menos un mes desde su recolección, pero no se encuentra información relativa a los cambios durante el envejecimiento natural (Strelec, Ugarcic-Hardi, Jurkovic y Jurkovic, 2007) por lo que no hay sustento bibliográfico para esta afirmación.

Posner y Deyoe (1986) han encontrado amplias fluctuaciones en las características de molienda en trigos recién cosechados, por ejemplo, un aumento de la extracción de harina de 2 a $5 \%$ en cinco meses de almacenamiento. La aplicación de métodos estadísticos reveló que este comportamiento no fue lineal.

El objetivo de este trabajo fue estudiar los cambios producidos en la calidad industrial de trigo candeal recién cosechado y durante siete meses de almacenamiento en condiciones similares a una explotación agropecuaria de la zona.

\section{MATERIALES Y MÉTODOS}

El ensayo se realizó sobre dos campañas de trigo candeal (2013-2014 y 2015-2016), en ambos casos la variedad utilizada fue Bonaerense INTA Cariló. Luego de la cosecha, los granos se almacenaron en un silo de chapa moldeada de 180 tn de capacidad en instalaciones de la Chacra 
Experimental Integrada Barrow (Tres Arroyos $38^{\circ}$ $\left.20^{\prime} \mathrm{S}, 60^{\circ} 13^{\prime} \mathrm{O}\right)$

Los muestreos se realizaron extrayendo tres muestras de $1 \mathrm{~kg}$ de granos de trigo cada quince días durante siete meses. La toma de muestras se efectuó con un calador de granos para silos. En la campaña 2013-2014, el ensayo comenzó el 30/12/2013 y finalizó el 14/07/2014. En la campaña 2015-2016, comenzó el 28/12/2015 y finalizó el 05/07/2016.

\section{Análisis de calidad en grano}

Se determinaron los siguientes parámetros de calidad de grano: porcentaje de proteína y humedad, utilizando el método NIRS. Aparato DS 2500 (FOSS, Hillerod, Dinamarca). Peso de mil granos, evaluado según Norma IRAM 15.853 (IRAM, 2012). Rendimiento molinero: la molienda se efectuó en un molino experimental Bühler 202 (Bühler, Uzwil, Suiza), previo acondicionamiento de los granos, según Normas IRAM 15.854- I y II (IRAM, 2014).

\section{Análisis de calidad en la sémola}

Para el análisis de calidad de sémola, se determinaron los siguientes parámetros: color, parámetros $L^{*}, a^{*}$ y $b^{*}$ utilizando el equipo Minolta Chromameter CR-310 (Konica Minolta, Tokio, Japón). Gluten húmedo, seco e índice de gluten con el equipo Glutomatic (Perten, Hagersten, Suecia) y según la Norma IRAM 15.864-II (IRAM, 2013). Farinograma, para el cual se usó una adaptación de la técnica de Ivine, Badley y Martin (Cereal Chemistry, Vol. 38, № 2, 1961), en la que el porcentaje de absorción de agua es constante (45 $\%)$ y el tiempo de amasado es fijo (8 min); se usó la amasadora de $50 \mathrm{~g}$ (Brabender OHG, Duisburg, Alemania). Por último se midió la actividad alfaamilásica, a través del índice de caída (falling number), según Norma IRAM 15.862 (IRAM, 2003). Para esto, se utilizó el equipo Falling Number Mod. 1400 (Perten, Hagersten, Suecia).

\section{Tratamiento estadístico}

Cada muestreo fue realizado por triplicado y analizado según análisis de la varianza (ANOVA simple), utilizando el test de Tukey para la comparación de medias, con un $p<0,05$. También se realizó la evaluación de las regresiones (lineales y no lineales) entre los momentos de muestreo y cada una de las variables de calidad medidas. Para esto se utilizó el paquete estadístico InfoStat (Di Rienzo et al., 2014).

\section{RESULTADOSY DISCUSIÓN}

\section{Análisis de calidad de grano}

\section{Campaña 2013-2014}

Los datos obtenidos en esta campaña corresponden a un trigo de bajo peso de granos (valores entre 35,9 y $32,8 \mathrm{~g})$ y buen contenido de proteína (11,8-10,9\%) (Tabla 1). Este último parámetro del grano no mostró una tendencia de cambio marcada durante los días de almacenamiento (Figura 1B). Mientras que el peso de los granos mostró diferencias estadísticas entre los muestreos (Tabla 1), pero sin evidenciar una tendencia a lo largo del tiempo de almacenamiento (Figura 1C).

El grano se cosechó seco y se mantuvo por debajo del 10,0\% de humedad hasta fines de marzo. Alcanzó un pico máximo de $14,4 \%$ a principios de septiembre (180 días desde la cosecha), pero descendió rápidamente. Estos valores muestran que el trigo se almacenó en buenas condiciones durante los meses que duró el ensayo (Tabla 1, Figura 1A).

Tabla 1. Determinaciones en grano realizadas a diferentes días desde la cosecha (DDC). Campaña 2013-2014.

\begin{tabular}{ccccc}
\hline DDC & PMG & PROT & HUM & REND \\
\hline $\mathbf{1 5}$ & $34,9 \mathrm{cde}$ & $11,0 \mathrm{a}$ & $10,0 \mathrm{a}$ & $65,8 \mathrm{a}$ \\
$\mathbf{3 0}$ & $\mathrm{s} / \mathrm{d}$ & $11,6 \mathrm{cdef}$ & $9,5 \mathrm{a}$ & $64,0 \mathrm{ab}$ \\
$\mathbf{4 5}$ & $32,8 \mathrm{a}$ & $11,7 \mathrm{def}$ & $9,3 \mathrm{a}$ & $66,2 \mathrm{a}$ \\
$\mathbf{6 0}$ & $35,9 \mathrm{e}$ & $11,4 \mathrm{bcd}$ & $9,8 \mathrm{a}$ & $65,8 \mathrm{a}$ \\
$\mathbf{7 5}$ & $33,3 \mathrm{abc}$ & $11,7 \mathrm{ef}$ & $9,4 \mathrm{a}$ & $69,7 \mathrm{~cd}$ \\
$\mathbf{9 0}$ & $34,6 \mathrm{bcde}$ & $11,6 \mathrm{cdef}$ & $9,8 \mathrm{a}$ & $69,3 \mathrm{bc}$ \\
$\mathbf{1 0 5}$ & $33,2 \mathrm{ab}$ & $11,7 \mathrm{ef}$ & $10,8 \mathrm{~b}$ & $66,7 \mathrm{bcd}$ \\
$\mathbf{1 2 0}$ & $34,0 \mathrm{abcd}$ & $11,6 \mathrm{cdef}$ & $11,8 \mathrm{c}$ & $72,2 \mathrm{def}$ \\
$\mathbf{1 3 5}$ & $35,4 \mathrm{de}$ & $11,8 \mathrm{f}$ & $11,8 \mathrm{c}$ & $71,1 \mathrm{cde}$ \\
$\mathbf{1 5 0}$ & $35,2 \mathrm{de}$ & $11,4 \mathrm{bc}$ & $13,3 \mathrm{c}$ & $72,5 \mathrm{ef}$ \\
$\mathbf{1 6 5}$ & $34,1 \mathrm{abcd}$ & $11,5 \mathrm{cdef}$ & $12,2 \mathrm{c}$ & $71,9 \mathrm{cde}$ \\
$\mathbf{1 8 0}$ & $35,5 \mathrm{de}$ & $10,9 \mathrm{a}$ & $14,4 \mathrm{e}$ & $71,8 \mathrm{bc}$ \\
$\mathbf{1 9 5}$ & $34,5 \mathrm{bcde}$ & $11,5 \mathrm{~b} \mathrm{cde}$ & $12,3 \mathrm{c}$ & $72,1 \mathrm{bc}$ \\
$\mathbf{2 1 0}$ & $33,4 \mathrm{abc}$ & $11,2 \mathrm{ab}$ & $9,5 \mathrm{a}$ & $71,9 \mathrm{f}$ \\
\hline
\end{tabular}

PMG: Peso de mil granos (g); PROT: proteína (porcentaje, base 13,5\% humedad); HUM: humedad (porcentaje); REND: rendimiento de sémola (porcentaje). s/d: sin dato.

Cada valor corresponde al promedio de tres repeticiones. Valores en una columna seguidos por la misma letra no son significativamente diferentes $(p<0,05)$.

Se observan fluctuaciones en la extracción de sémola al comienzo del almacenamiento: desde $65,8 \%$ al inicio del muestreo hasta alcanzar un rendimiento mayor al $71,0 \%$ entre los 120 y 150 días desde la cosecha (en adelante, DDC); a partir de ese momento, la curva se estabiliza (Tabla 1, 


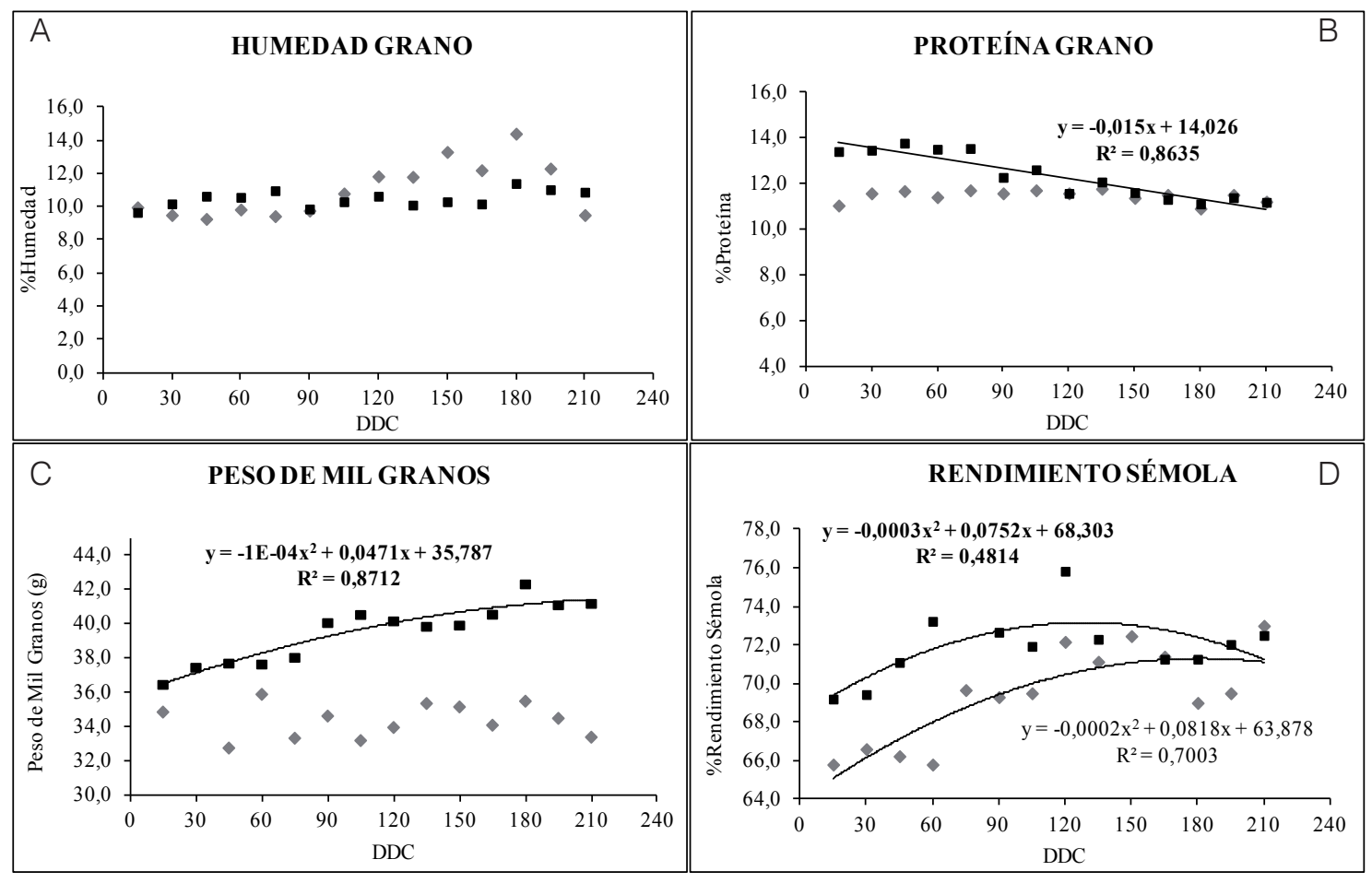

Figura 1. Relación entre los parámetros de calidad de grano: humedad de grano; proteína del grano; peso de mil granos (g) y porcentaje de rendimiento de sémola; en función de los días desde la cosecha (DDC), para todo el periodo evaluado: campaña $2013-2014$ ( $\downarrow$ ) y campaña 2015-2016 ( $\boldsymbol{\bullet}$, ecuaciones en negrita).

Figura 1D). Estos resultados coinciden con los obtenidos por Posner y Deyoe en 1986 para el comportamiento molinero de muestras de trigo pan en condiciones ambientales controladas. Y resulta opuesto a lo expuesto por Lukow y colaboradores en 1995, quienes hallaron en trigo pan una leve disminución del rendimiento de harina durante 15 meses de almacenamiento.

\section{Campaña 2015-2016}

En esta campaña, el análisis de la calidad de grano mostró valores de peso de mil más altos que en la campaña anterior (promedio del ensayo 39,5 g) (Tabla 2, Figura 1C), posiblemente debido a condiciones desfavorables de alta temperatura durante el llenado de grano en la campaña 20132014 (Larsen y Jensen, 2014). En este caso, los valores fueron en aumento a lo largo de los días de almacenamiento (Tabla 2). Esto se encuentra íntimamente ligado al aumento en el rendimiento de sémola que se evidencia hasta los 120 DDC (Figura 1D), ya que existe una estrecha correlación positiva entre peso de mil granos (PMG) y rendimiento de sémola (Matsuo y Dexter, 1980; Seghezzo, 2014). En este ensayo, la correlación obtenida entre estos parámetros de calidad fue de 0,63. No se encontró relación similar en la campaña anterior, lo cual pudo deberse a las condiciones climáticas anteriormente mencionadas en la campaña 20132014.

Tabla 2. Determinaciones en grano realizadas a diferentes días desde la cosecha (DDC). Campaña 2015-2016.

\begin{tabular}{ccccc}
\hline DDC & PMG & PROT & HUM & REND \\
\hline $\mathbf{1 5}$ & $36,45 \mathrm{a}$ & $13,40 \mathrm{e}$ & $9,66 \mathrm{a}$ & $69,20 \mathrm{ab}$ \\
$\mathbf{3 0}$ & $37,45 \mathrm{ab}$ & $13,46 \mathrm{e}$ & $10,17 \mathrm{bc}$ & $69,43 \mathrm{abc}$ \\
$\mathbf{4 5}$ & $37,70 \mathrm{ab}$ & $13,77 \mathrm{e}$ & $10,63 \mathrm{~cd}$ & $71,10 \mathrm{abcd}$ \\
$\mathbf{6 0}$ & $37,64 \mathrm{ab}$ & $13,50 \mathrm{e}$ & $10,57 \mathrm{~cd}$ & $73,23 \mathrm{abcd}$ \\
$\mathbf{7 5}$ & $38,03 \mathrm{~b}$ & $13,53 \mathrm{e}$ & $10,97 \mathrm{de}$ & $\mathrm{s} / \mathrm{d}$ \\
$\mathbf{9 0}$ & $40,06 \mathrm{c}$ & $12,27 \mathrm{~cd}$ & $9,87 \mathrm{ab}$ & $72,67 \mathrm{cde}$ \\
$\mathbf{1 0 5}$ & $40,53 \mathrm{c}$ & $12,60 \mathrm{~d}$ & $10,30 \mathrm{bc}$ & $71,93 \mathrm{bcd}$ \\
$\mathbf{1 2 0}$ & $40,16 \mathrm{c}$ & $11,57 \mathrm{abc}$ & $10,63 \mathrm{~cd}$ & $75,83 \mathrm{e}$ \\
$\mathbf{1 3 5}$ & $39,84 \mathrm{c}$ & $12,07 \mathrm{bcd}$ & $10,10 \mathrm{abc}$ & $72,30 \mathrm{bcd}$ \\
$\mathbf{1 5 0}$ & $39,91 \mathrm{c}$ & $11,60 \mathrm{abc}$ & $10,30 \mathrm{bc}$ & $\mathrm{s} / \mathrm{d}$ \\
$\mathbf{1 6 5}$ & $40,55 \mathrm{c}$ & $11,30 \mathrm{a}$ & $10,17 \mathrm{abc}$ & $71,27 \mathrm{abcd}$ \\
$\mathbf{1 8 0}$ & $42,32 \mathrm{~d}$ & $11,10 \mathrm{a}$ & $11,40 \mathrm{e}$ & $71,27 \mathrm{abcd}$ \\
$\mathbf{1 9 5}$ & $41,10 \mathrm{~cd}$ & $11,38 \mathrm{ab}$ & $11,03 \mathrm{de}$ & $72,03 \mathrm{bcd}$ \\
$\mathbf{2 1 0}$ & $41,2 \mathrm{~cd}$ & $11,20 \mathrm{a}$ & $10,9 \mathrm{de}$ & $72,5 \mathrm{bcde}$ \\
\hline
\end{tabular}

PMG: peso de mil granos (g); PROT: proteína (porcentajes, base 13,5\% humedad); HUM: humedad (porcentaje); REND: rendimiento de Sémola (porcentaje). s/d: sin dato.

Cada valor corresponde al promedio de tres repeticiones. Valores en una columna seguidos por la misma letra no son significativamente diferentes $(p<0,05)$. 
Al igual que en el año anterior, el contenido de humedad del grano se ubicó en valores normales durante el tiempo que duró el ensayo $(<11,5 \%)$ (Tabla 2, Figura 1A).

El contenido de proteína del grano mostró una disminución de $0,015 \%$ en cada muestreo, y pasó de un contenido de $13,4 \%$ al inicio a un $11,2 \%$ al final (Tabla 2, Figura 1B).

\section{Análisis de calidad de sémola}

\section{Campaña 2013-2014}

En la sémola obtenida se examinó la actividad alfa-amilásica mediante el test Falling Number (FN), y se obtuvieron valores entre 521 y 821 segundos (Tabla 3, Figura 2A). Como era de esperar, el valor de FN aumentó durante el período muestreado debido al proceso natural de disminución de la actividad de la enzima alfa-amilasa (Campaña y Sempé, 1980; Lukow, White y Sinha, 1995). Los valores de FN hallados fueron elevados, poniendo de manifiesto la baja actividad alfa-amilásica de esta campaña.

Tabla 3. Determinaciones en sémola, realizadas a diferentes días desde la cosecha (DDC). Campaña 2013-2014.

\begin{tabular}{ccccc}
\hline & & \multicolumn{3}{c}{ COLOR } \\
\cline { 3 - 5 } DDC & FN & $\mathbf{L}^{*}$ & $\mathbf{a}^{*}$ & $\mathbf{b}^{*}$ \\
\hline $\mathbf{1 5}$ & $545 \mathrm{a}$ & $82,97 \mathrm{a}$ & $-3,30 \mathrm{bcde}$ & $22,90 \mathrm{~b}$ \\
$\mathbf{3 0}$ & $521 \mathrm{ab}$ & $84,05 \mathrm{bc}$ & $-3,33 \mathrm{bcd}$ & $23,20 \mathrm{bc}$ \\
$\mathbf{4 5}$ & $576 \mathrm{bcd}$ & $83,90 \mathrm{~b}$ & $-3,22 \mathrm{cdef}$ & $23,24 \mathrm{bc}$ \\
$\mathbf{6 0}$ & $562 \mathrm{abc}$ & $85,90 \mathrm{~h}$ & $-3,78 \mathrm{a}$ & $25,07 \mathrm{f}$ \\
$\mathbf{7 5}$ & $567 \mathrm{abcd}$ & $84,42 \mathrm{bcd}$ & $-3,15 \mathrm{ef}$ & $23,59 \mathrm{~cd}$ \\
$\mathbf{9 0}$ & $569 \mathrm{abcd}$ & $84,54 \mathrm{cde}$ & $-3,21 \mathrm{def}$ & $23,12 \mathrm{bc}$ \\
$\mathbf{1 0 5}$ & $621 \mathrm{de}$ & $84,18 \mathrm{bc}$ & $-3,74 \mathrm{a}$ & $23,70 \mathrm{~cd}$ \\
$\mathbf{1 2 0}$ & $774 \mathrm{f}$ & $84,32 \mathrm{bc}$ & $-3,25 \mathrm{cdef}$ & $23,59 \mathrm{~cd}$ \\
$\mathbf{1 3 5}$ & $665 \mathrm{e}$ & $85,87 \mathrm{gh}$ & $-3,77 \mathrm{a}$ & $25,14 \mathrm{f}$ \\
$\mathbf{1 5 0}$ & $619 \mathrm{de}$ & $84,12 \mathrm{bc}$ & $-3,22 \mathrm{cdef}$ & $23,47 \mathrm{bcd}$ \\
$\mathbf{1 6 5}$ & $611 \mathrm{cde}$ & $85,10 \mathrm{ef}$ & $-3,39 \mathrm{bc}$ & $23,72 \mathrm{~cd}$ \\
$\mathbf{1 8 0}$ & $616 \mathrm{cde}$ & $85,27 \mathrm{fg}$ & $-3,45 \mathrm{~b}$ & $23,89 \mathrm{de}$ \\
$\mathbf{1 9 5}$ & $651 \mathrm{e}$ & $83,07 \mathrm{a}$ & $-3,34 \mathrm{bcd}$ & $24,40 \mathrm{e}$ \\
$\mathbf{2 1 0}$ & $821 \mathrm{f}$ & $84,94 \mathrm{def}$ & $-3,13 \mathrm{f}$ & $21,90 \mathrm{a}$ \\
\hline
\end{tabular}

FN: falling number (seg); parámetros de color: $L^{*}$ : luminosidad; $a^{*}$ : verde; $b^{*}$ : amarillo

Cada valor corresponde al promedio de tres repeticiones. Valores en una columna seguidos por la misma letra no son significativamente diferentes $(p<0,05)$.

Los parámetros de color, aunque presentaron diferencias estadísticas entre los muestreos, no marcaron tendencias a lo largo del ensayo (Tabla 3). El parámetro b* (representado en la Figura 2B), que indica la coloración amarilla, es el que se usa para caracterizar sémolas y evaluar su aptitud en la elaboración de pastas (Seghezzo, 2014).
El contenido de gluten húmedo estuvo entre 27,3 y 30,3\%; y el de gluten seco entre 8,7 y $9,7 \%$. Aunque se hallaron diferencias estadísticas entre los muestreos (Tabla 4), no marcaron tendencias claras durante el tiempo que duro el ensayo (Figuras 2C y 2D). Esto está en estrecha relación con el contenido de proteína que tampoco mostró un ajuste claro con los DDC (Tabla 1, Figura 1A). Algunos autores observaron una disminución no significativa en el contenido de gluten al cabo de algunos meses de almacenamiento (Campaña y Sempé, 1980; Miravalles, Alonso y Zecca., 2008; Gyimes, Szabó y Veha, 2011).

En cuanto a la calidad del gluten, medida a través del parámetro gluten index, se observa que se mantiene estable durante el ensayo, con un promedio de $87 \%$ (Tabla 4, Figura 2E). Este valor se considera óptimo para el trigo, por encontrarse en el rango de $\mathrm{Gl}>70 \%$ (Cubadda, Carcea, Pasqui y Perten, 1991; Borkowska, Grundas y Styk., 1999).

Tabla 4. Determinaciones en sémola, realizadas a diferentes días desde la cosecha (DDC). Campaña 2013-2014.

\begin{tabular}{cccccc}
\hline \multicolumn{3}{c}{ GLUTEN } & \multicolumn{3}{c}{ FARINOGRAMA } \\
\hline DDC & GH & GI & GS & NE & AFLO \\
\hline $\mathbf{1 5}$ & $28,4 \mathrm{bcde}$ & $81 \mathrm{cde}$ & $9,2 \mathrm{bc}$ & $25,4 \mathrm{a}$ & $22 \mathrm{a}$ \\
$\mathbf{3 0}$ & $28,8 \mathrm{cdef}$ & $73 \mathrm{ab}$ & $9,3 \mathrm{c}$ & $35,0 \mathrm{e}$ & $22 \mathrm{a}$ \\
$\mathbf{4 5}$ & $27,8 \mathrm{ab}$ & $88 \mathrm{fg}$ & $9,0 \mathrm{~b}$ & $35,2 \mathrm{e}$ & $24 \mathrm{abcd}$ \\
$\mathbf{6 0}$ & $28,4 \mathrm{bcde}$ & $84 \mathrm{def}$ & $9,2 \mathrm{bc}$ & $34,3 \mathrm{de}$ & $24 \mathrm{abcd}$ \\
$\mathbf{7 5}$ & $28,1 \mathrm{bc}$ & $78 \mathrm{bc}$ & $9,2 \mathrm{bc}$ & $31,3 \mathrm{bc}$ & $23 \mathrm{ab}$ \\
$\mathbf{9 0}$ & $28,8 \mathrm{cdef}$ & $96 \mathrm{~h}$ & $9,3 \mathrm{c}$ & $31,2 \mathrm{bc}$ & $28 \mathrm{e}$ \\
$\mathbf{1 0 5}$ & $29,4 \mathrm{f}$ & $80 \mathrm{~cd}$ & $9,3 \mathrm{c}$ & $30,6 \mathrm{~b}$ & $24 \mathrm{abc}$ \\
$\mathbf{1 2 0}$ & $28,9 \mathrm{def}$ & $86 \mathrm{efg}$ & $9,1 \mathrm{bc}$ & $32,9 \mathrm{bcde}$ & $26 \mathrm{cde}$ \\
$\mathbf{1 3 5}$ & $29,1 \mathrm{ef}$ & $87 \mathrm{fg}$ & $9,3 \mathrm{c}$ & $32,6 \mathrm{bcde}$ & $25 \mathrm{abcde}$ \\
$\mathbf{1 5 0}$ & $28,5 \mathrm{bcde}$ & $91 \mathrm{gh}$ & $9,2 \mathrm{bc}$ & $32,7 \mathrm{bcde}$ & $26 \mathrm{cde}$ \\
$\mathbf{5}$ & $30,3 \mathrm{~g}$ & $72 \mathrm{a}$ & $9,7 \mathrm{~d}$ & $34,0 \mathrm{cde}$ & $27 \mathrm{de}$ \\
$\mathbf{1 8 0}$ & $29,0 \mathrm{ef}$ & $87 \mathrm{fg}$ & $9,3 \mathrm{c}$ & $32,6 \mathrm{bcde}$ & $26 \mathrm{bcde}$ \\
$\mathbf{1 9 5}$ & $28,2 \mathrm{bcd}$ & $84 \mathrm{cdef}$ & $9,0 \mathrm{~b}$ & $32,0 \mathrm{bcd}$ & $24 \mathrm{abcd}$ \\
$\mathbf{2 1 0}$ & $27,3 \mathrm{a}$ & $84 \mathrm{def}$ & $8,7 \mathrm{a}$ & $30,8 \mathrm{~b}$ & $25 \mathrm{abcde}$ \\
\hline
\end{tabular}

$\mathrm{GH}$ : gluten húmedo (porcentaje); GS: gluten seco (porcentaje); Gl: índice de gluten (porcentaje); farinograma-NE: nivel de energía y AFLO (porcentaje): aflojamiento.

Cada valor corresponde al promedio de tres repeticiones. Valores en una columna seguidos por la misma letra no son significativamente diferentes $(p<0,05)$.

La evaluación de la reología de las masas, a través del farinógrafo, mostró que no se evidenciaron tendencias a lo largo de los siete meses de almacenamiento (Tabla 4, Figuras 2F y 2G).

\section{Campaña 2015-2016}

El análisis de FN de esta campaña reafirmó lo obtenido en 2013-2014 y lo citado por Campaña y Sempé en 1980, aunque en este caso la disminución con el tiempo de almacenamiento fue leve. 

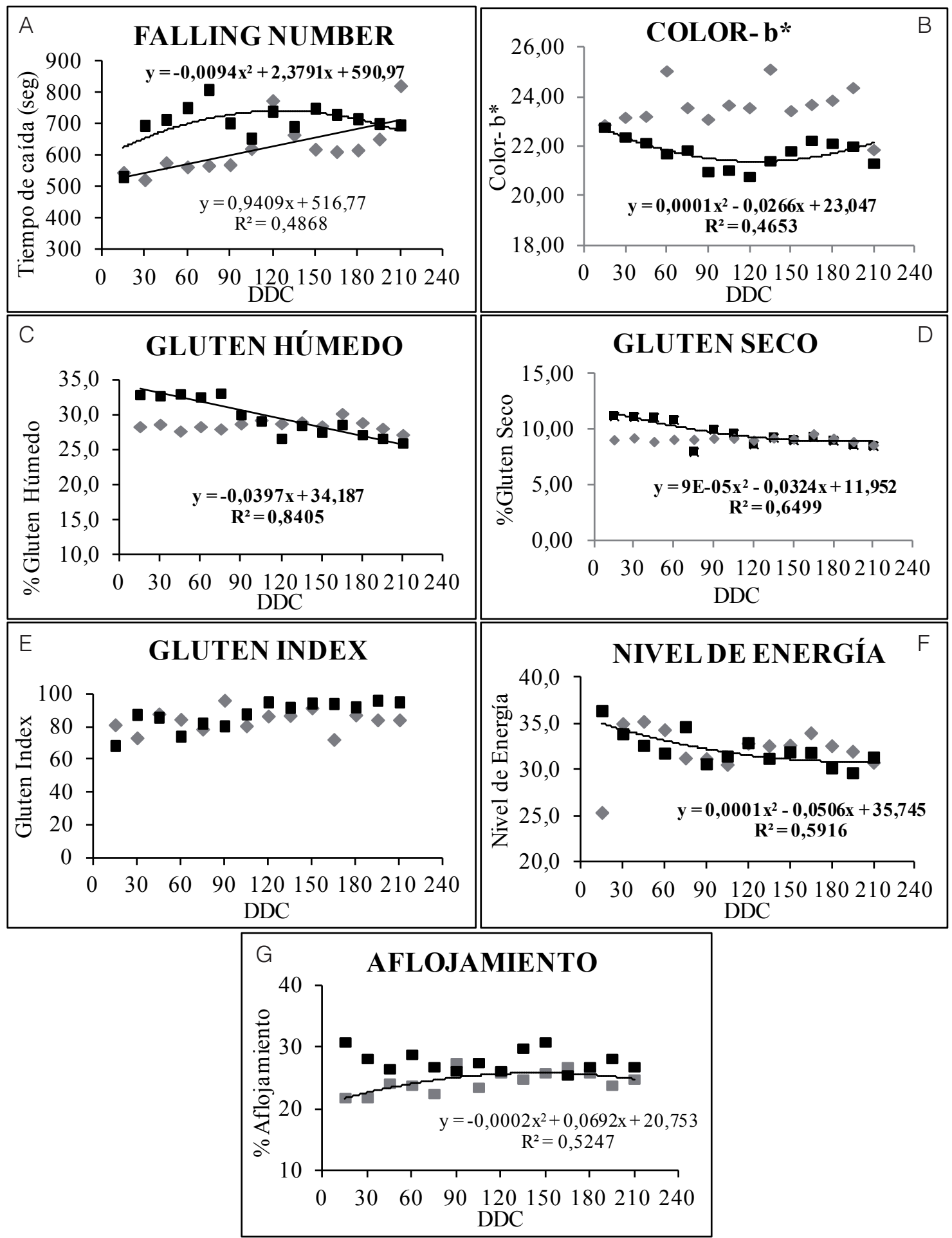

Figura 2. Relación entre los parámetros de calidad de sémola: falling number (FN, seg); Color-b*; contenido de gluten húmedo (GH, porcentaje); contenido de gluten seco (GS, porcentaje); gluten índex (GI); nivel de energía (NE) y aflojamiento (AFLO, porcentaje), en función de los días desde la cosecha (DDC), para todo el período evaluado: campaña 2013-2014 (») y campaña 2015-2016 (ロ, ecuaciones en negrita). 
Los valores de FN resultaron elevados debido a una baja actividad alfa-amilásica, generada por las condiciones de baja humedad ambiente durante la cosecha del cultivo (Tabla 5, Figura 2A)

Tabla 5. Determinaciones en sémola, realizadas a diferentes días desde la cosecha (DDC). Campaña 2015-2016.

\begin{tabular}{ccccc}
\hline & & \multicolumn{3}{c}{ COLOR } \\
\cline { 3 - 5 } DDC & FN & $\mathbf{L}^{*}$ & $\mathbf{a}^{*}$ & $\mathbf{b}^{*}$ \\
\hline $\mathbf{1 5}$ & $531 \mathrm{a}$ & $84,82 \mathrm{cde}$ & $-3,54 \mathrm{ab}$ & $22,80 \mathrm{f}$ \\
$\mathbf{3 0}$ & $695 \mathrm{bc}$ & $84,67 \mathrm{~cd}$ & $-3,35 \mathrm{bc}$ & $22,41 \mathrm{ef}$ \\
$\mathbf{4 5}$ & $713 \mathrm{bc}$ & $84,91 \mathrm{cde}$ & $-3,38 \mathrm{bc}$ & $22,18 \mathrm{cdef}$ \\
$\mathbf{6 0}$ & $751 \mathrm{~cd}$ & $85,29 \mathrm{def}$ & $-3,41 \mathrm{bc}$ & $21,74 \mathrm{bcde}$ \\
$\mathbf{7 5}$ & $809 \mathrm{~d}$ & $85,16 \mathrm{cdef}$ & $-3,39 \mathrm{bc}$ & $21,88 \mathrm{bcdef}$ \\
$\mathbf{9 0}$ & $702 \mathrm{bc}$ & $85,41 \mathrm{efg}$ & $-3,39 \mathrm{bc}$ & $21,01 \mathrm{ab}$ \\
$\mathbf{1 0 5}$ & $654 \mathrm{~b}$ & $85,57 \mathrm{fg}$ & $-3,57 \mathrm{ab}$ & $21,07 \mathrm{ab}$ \\
$\mathbf{1 2 0}$ & $740 \mathrm{bcd}$ & $85,30 \mathrm{def}$ & $-3,27 \mathrm{~cd}$ & $20,82 \mathrm{a}$ \\
$\mathbf{1 3 5}$ & $691 \mathrm{bc}$ & $85,64 \mathrm{fg}$ & $-3,45 \mathrm{bc}$ & $21,45 \mathrm{abcd}$ \\
$\mathbf{1 5 0}$ & $749 \mathrm{~cd}$ & $84,81 \mathrm{cde}$ & $-3,74 \mathrm{a}$ & $21,85 \mathrm{bcde}$ \\
$\mathbf{1 6 5}$ & $730 \mathrm{bcd}$ & $82,62 \mathrm{a}$ & $-3,10 \mathrm{~d}$ & $22,27 \mathrm{def}$ \\
$\mathbf{1 8 0}$ & $716 \mathrm{bc}$ & $86,03 \mathrm{~g}$ & $-3,46 \mathrm{bc}$ & $22,15 \mathrm{cdef}$ \\
$\mathbf{1 9 5}$ & $701 \mathrm{bc}$ & $84,52 \mathrm{c}$ & $-3,53 \mathrm{ab}$ & $22,04 \mathrm{cdef}$ \\
$\mathbf{2 1 0}$ & $696 \mathrm{bc}$ & $83,90 \mathrm{~b}$ & $-3,40 \mathrm{bc}$ & $21,40 \mathrm{abc}$ \\
\hline
\end{tabular}

FN: falling number (seg); parámetros de color: $L^{*}$ : luminosidad; $a^{*}$ : verde; $b^{*}$ : amarillo.

Cada valor corresponde al promedio de tres repeticiones. Valores en una columna seguidos por la misma letra no son significativamente diferentes $(p<0,05)$.

Los parámetros de color, al igual que en la campaña analizada anteriormente, no mostraron tendencias importantes a lo largo del ensayo (Tabla 5). En la Figura 2B está representado el parámetro $\mathrm{b}^{*}$ que, como fue mencionado anteriormente, tiene gran importancia en la elaboración de pastas. En esta figura puede verse cómo hasta los 120 DDC se evidenció un descenso de dos puntos, pasando de 22,80 (15 DDC) a 20,82 (120 DDC), y luego comenzó a aumentar hasta el final del ensayo, donde se registraron valores cercanos a 22,00.

En cuanto a la calidad y cantidad de gluten, se observó que el contenido de gluten húmedo tuvo una disminución leve a lo largo de los muestreos, a razón de 0,04\%, pasando de un contenido de 33,0 a un valor de $26,1 \%$ (Tabla 6, Figura 2C). Esta disminución está íntimamente ligada a la disminución observada en el contenido de proteína (Tabla 2. Figura 1B). El gluten seco también disminuye, en forma menos marcada (Tabla 6, Figura 2D), y el gluten índex aumenta (Tabla 6, Figura 2E), lo que estaría indicando que los glútenes tienden a ser más fuertes, en coincidencia con lo expuesto por Miravalles y colaboradores en 2008. En la bibliografía se ha vinculado este tipo de respuesta con la ocurrencia, a nivel molecular, de un aumento en la
Tabla 6. Determinaciones en sémola, realizadas a diferentes días desde la cosecha (DDC). Campaña 2015-2016.

\begin{tabular}{cccccc}
\hline \multicolumn{3}{c}{ GLUTEN } & \multicolumn{3}{c}{ FARINOGRAMA } \\
\hline DDC & GH & GI & SECO & NE & AFLO \\
\hline $\mathbf{1 5}$ & $33,00 \mathrm{f}$ & $68 \mathrm{a}$ & $11,3 \mathrm{~g}$ & $36,4 \mathrm{f}$ & $31 \mathrm{~d}$ \\
$\mathbf{3 0}$ & $32,83 \mathrm{f}$ & $87 \mathrm{ef}$ & $11,2 \mathrm{~g}$ & $33,9 \mathrm{de}$ & $28 \mathrm{abcd}$ \\
$\mathbf{4 5}$ & $33,10 \mathrm{f}$ & $86 \mathrm{de}$ & $11,2 \mathrm{~g}$ & $32,6 \mathrm{~cd}$ & $27 \mathrm{ab}$ \\
$\mathbf{6 0}$ & $32,67 \mathrm{f}$ & $74 \mathrm{~b}$ & $11,0 \mathrm{~g}$ & $31,8 \mathrm{bc}$ & $29 \mathrm{bcd}$ \\
$\mathbf{7 5}$ & $33,20 \mathrm{f}$ & $82 \mathrm{~cd}$ & $8,1 \mathrm{a}$ & $34,7 \mathrm{ef}$ & $27 \mathrm{abc}$ \\
$\mathbf{9 0}$ & $30,13 \mathrm{e}$ & $80 \mathrm{c}$ & $10,1 \mathrm{f}$ & $30,6 \mathrm{ab}$ & $26 \mathrm{ab}$ \\
$\mathbf{1 0 5}$ & $29,23 \mathrm{de}$ & $88 \mathrm{efg}$ & $9,7 \mathrm{ef}$ & $31,5 \mathrm{abc}$ & $28 \mathrm{abc}$ \\
$\mathbf{1 2 0}$ & $26,77 \mathrm{ab}$ & $95 \mathrm{~h}$ & $8,8 \mathrm{bc}$ & $32,9 \mathrm{cde}$ & $26 \mathrm{ab}$ \\
$\mathbf{1 3 5}$ & $28,60 \mathrm{~cd}$ & $92 \mathrm{fgh}$ & $9,4 \mathrm{de}$ & $31,2 \mathrm{abc}$ & $30 \mathrm{~cd}$ \\
$\mathbf{1 5 0}$ & $27,63 \mathrm{bc}$ & $94 \mathrm{~h}$ & $9,1 \mathrm{~cd}$ & $31,9 \mathrm{bc}$ & $31 \mathrm{~d}$ \\
$\mathbf{1 6 5}$ & $28,73 \mathrm{~cd}$ & $94 \mathrm{~h}$ & $9,4 \mathrm{de}$ & $31,9 \mathrm{bc}$ & $26 \mathrm{a}$ \\
$\mathbf{1 8 0}$ & $27,30 \mathrm{~b}$ & $92 \mathrm{gh}$ & $9,1 \mathrm{~cd}$ & $30,2 \mathrm{ab}$ & $27 \mathrm{abc}$ \\
$\mathbf{1 9 5}$ & $26,77 \mathrm{ab}$ & $96 \mathrm{~h}$ & $8,7 \mathrm{bc}$ & $29,7 \mathrm{a}$ & $28 \mathrm{abcd}$ \\
$\mathbf{2 1 0}$ & $26,1 \mathrm{a}$ & $95 \mathrm{~h}$ & $8,6 \mathrm{~b}$ & $31,4 \mathrm{abc}$ & $27 \mathrm{abc}$ \\
\hline
\end{tabular}

GH: gluten húmedo (porcentaje); GS: gluten seco (porcentaje); Gl: índice de gluten (porcentaje). Farinograma- NE: nivel de energía; AFLO (porcentaje): aflojamiento.

Cada valor corresponde al promedio de tres repeticiones. Valores en una columna seguidos por la misma letra no son significativamente diferentes $(p<0,05)$.

fracción insoluble de las gluteninas del endosperma (Gras y O'Riordan, 1998).

Los parámetros reológicos analizados, al igual que en la campaña 2013-2014, no mostraron cambios de importancia durante el tiempo que duró el almacenamiento. Estos resultados son considerados positivos puesto que puede asegurarse el mantenimiento de las características reológicas del trigo candeal durante siete meses de almacenamiento, en las condiciones en que fueron llevados dichos ensayos.

\section{CONCLUSIONES}

Los resultados indican que mientras el trigo se mantenga en condiciones adecuadas, seco y sano, no hay evidencia de variaciones significativas en los parámetros de calidad medidos excepto para la extracción molinera que muestra una tendencia creciente hasta los 120-150 días desde la cosecha, lo cual podría resultar interesante desde el punto de vista económico.

En lo que hace a la calidad de sémola, y como ha sido citado por otros autores, la actividad alfaamilásica tiende a aumentar con el tiempo de almacenamiento, como un proceso natural del grano. En el caso de la cantidad de gluten, se puede decir que existe una leve disminución con el tiempo de almacenamiento, mientras que la calidad de 
ese gluten aumenta dando masas más fuertes. De todas maneras, esta variación no llega a traducirse en cambios importantes de la reología de las masas.

Por lo expuesto anteriormente es que podría resultar beneficioso mezclar trigo viejo con trigo nuevo y así, minimizar las fluctuaciones en las características molineras de la sémola durante su almacenamiento poscosecha.

\section{BIBLIOGRAFÍA}

Borkowska, H., Grundas, S. y Styk, B.(1999). Influence of nitrogen fertilization of winter wheat on its gluten quality. Int. Agrophysics, 13, 333-335.

Campaña, L. E. y Sempé, M. E. (1980). Almacenamiento de trigo: variación de su calidad. Revista de la Facultad de Agronomía, Tomo LVI, Entrega 1-2.

Cubadda, R., Carcea, M., Pasqui, L. A. y Perten, H. (1991). Metodorapido di valutazione della qualità del glutine delle semole e del grano duro. Chimica dei Cereali. Tecnicamolitoria, 861-874.

Di Rienzo, J. A., Casanoves, F., Balzarini, M. G., Gonzalez, I., Tablada, M. y Robledo, C. W. (2014). InfoStat, versión 2014, Grupo InfoStat, FCA, Universidad Nacional de Córdoba, Argentina.

Gras P. W. y O'Riordan, B. (1998). Why wheat quality changes in storage. En Proceecings of the first. Australian Postharvest Technical Conference. Canberra, 2003.

Gyimes, E., Szabó, B. y Veha, A. (2011). Changing of Wheat Quality Parameters like Food Raw Material during Short Term Storage. (http://www.agir.ro/buletine/1277.pdf).

Irvine, G. M., Bradley J. W. y Martin, G. C. (1961). A farinograph technique for macoronidough. Cereal Chem, 38 (2), 153-164.

Larsen, A. O. y Jensen, C. A. (2014). Evaluación de cultivares de trigo candeal en Barrow - Campaña 20132014. Instituto Nacional de Tecnología Agropecuaria. Recuperado el 10 de septiembre de 2017 de http:// inta.gob.ar/documentos/evaluacion-de-cultivares-detrigo-candeal-en-barrow-campana-2013-2014

Lukow, O. M., White, N. D. G. y Sinha, R. N. (1995). Influence of Ambient Storage Conditions on the Breadmaking Quality of Two Hard Red Spring Wheats. Journal of Stored Products Research, 31(4), 279-289.

Matsuo, R. R. y Dexter, J. E. (1980). Relationship between some durum wheat physical characteristics and semolina milling proprieties. Canadian Journal of PlantScience, 60, 49-53.

Ministerio de Agroindustria de la Nación. Mercados Agroindustriales. Subsecretaría de Información y Estadística Pública. Árbol de series temporales. Recuperado el 22 de mayo de 2017 de http://dev.siia.gov.ar/series

Miravalles, M., Alonso, D. y Zecca, G. (2008). Cambios en la Calidad Industrial del Trigo para Fideos durante el Almacenamiento. VII Congreso Nacional de Trigo, Santa Rosa, Argentina.

Norma IRAM 15.853. Cereales. Método de determinación de la masa de 1000 granos. Instituto Argentino de Normalización y Certificación, Argentina, 31 de octubre de 2012

Norma IRAM 15.854-1. Trigo. Molienda experimental para trigo pan. Parte 1 - Preparación y acondicionamiento de la muestra, y Norma IRAM 15854-2. Trigo. Molienda experimental para trigo pan. Parte 2 - Método Bühler®. Instituto Argentino de Normalización y Certificación, Argentina, 20 de enero de 2014

Norma IRAM 15.862.Cereales. Determinación de la actividad de la alfa-amilasa (número de caída), según Hagberg-Perten. Instituto Argentino de Normalización y Certificación, Argentina, 20 de marzo de 2003.

Norma IRAM 15.864-2. Trigo y harina de trigo. Determinación de gluten húmedo, de gluten seco y de índice de gluten. Método de lavado automático. Parte 2 - Lavado con solución salina. Instituto Argentino de Normalización y Certificación, Argentina, 19 de abril de 2013.

Pomeranz, Y. (ed.) (1978). Wheat: Chemistry and Technology. Washington: AACC.

Posner E. S. y Deyoe, C. W. (1986). Changes in Milling Properties of Newly Harvested Hard Wheat During Storage. Cereal Chem, 63(5), 451-456.

Seghezzo M. L. (2014). Calidad en trigo candeal. Ediciones Instituto Nacional de Tecnología Agropecuaria, pp. 3-68.

Strelec, I., Ugarcic-Hardi, Z., Jurkovic, Z. y Jurkovic, V. (2007). Physicochemical Changes During Wheat Seeds Ageing at Different Storage Conditions. Trabajo presentado en el IV Congreso Internacional de harina y pan y VI Congreso Croata de tecnología de cereales, Croacia.

Swanson C. O. (1931). The Story of a Grain of Wheat From Reaper to Roll. Association of Operative Millers Bull., 370.

Swanson C. O. (1946). Effects of Rain on Wheat During Harvest. Agr. Exp. Station; Kansas University; Technical Bulluletin, 60 . 